\title{
Improved CNT-Si heterojunction solar cell with structured single-walled carbon nanotubes
}

\author{
Shigeo Maruyama ${ }^{1 *}$, Kehang Cui ${ }^{1}$, Takaaki Chiba ${ }^{1}$, Olivier Reynaud ${ }^{2}$, Shunjiro Fujii ${ }^{3}$, Albert \\ Nasibulin $^{2}$, Shohei Chiashi ${ }^{1}$, Hiriomichi Kataura ${ }^{3}$, Esko Kauppinen ${ }^{2}$ \\ ${ }^{1}$ Department of Mechanical Engineering, The University of Tokyo, Japan 113-8656 \\ ${ }^{2}$ Department of Applied Physics, Aalto University School of Science, Finland \\ ${ }^{3}$ Nanosystem Research Institute, National Institute of Advanced Industrial Science and Technology, \\ Japan \\ maruyama@photon.t.u-tokyo.ac.jp
}

Single-walled carbon nanotubes (SWNTs) with outstanding electronic, optical, mechanical, and thermal properties are expected to be the most promising materials for next-generation energy devices as well as optical and electronic devices. However, the structure controlled assembly of SWNTs for various devices is still challenging. In this study, we discuss two different SWNT assemblies for SWNT-Si heterojuction solar cells.

We proposed a water vapor treatment to build up SWNTs to a self-assembled micro-honeycomb network for the application of solar cells [1]. The microhoneycomb network consists of vertical aggregated SWNT walls and a buckypaper bottom (quasi 2 dimentional random network). This hierarchical structure exhibits lower sheet resistance and higher optical transmittance compared with the buckypaper. The heterojunction solar cell was fabricated by dry depositing the SWNT film to the $3 \mathrm{~mm}$ by $3 \mathrm{~mm}$ n-type silicon substrate. The pristine SWNT-Si heterojunction solar cell shows a recordhigh fill factor of $72 \%$ as well as a power conversion efficiency (PCE) of $6 \%$ without tuning the diameter or height of original vertically aligned SWNTs [2]. The PCE remains stable for months in ambient condition. A PCE exceeding $10 \%$ is achieved in the dry state after dilute nitric acid treatment.

On the other hand, heterojucntion solar cells using highly transparentconductive SWNT films from controlled bundle-diameter and long bundle length [3] are also promising. Here, SWNTs were synthesized by the thermal decomposition of ferrocene vapor in a carbon monoxide atmosphere, with the average diameter of approx. $2 \mathrm{~nm}$. The SWNT films showed a sheet resistance of $117 \Omega / \mathrm{sq}$. at the transmittance of $91 \%$ over the AM1.5G spectrum. Our preliminary test result shows the PCE of $10.12 \%$, with short-circuit current, open-circuit voltage and fill factor of $30.9 \mathrm{~mA} / \mathrm{cm}^{2}, 540 \mathrm{mV}$ and $60 \%$, respectively.

Both types of solar cells are stable after months, which is attributed to the high-purity pristine SWNTs. The updated performance of these solar cells and the detailed mechanism will be discussed.

References:

[1] K. Cui, T. Chiba, S. Omiya, T. Thurakitseree, P. Zhao, S. Fujii, H. Kataura, E. Einarsson, S. Chiashi, S. Maruyama, J. Phys. Chem. Lett., (2013), 4, 2571.

[2] Y. Murakami, S. Chiashi, Y. Miyauchi, M. Hu, M. Ogura, T. Okubo, S. Maruyama, Chem. Phys. Lett., (2004), 385, 298.

[3] A. G. Nasibulin, A. Kaskela, K. Mustonen, A. S. Anisimov, V. Ruiz, S. Kivistö, S. Rackauskas, M. Y. Timmermans, M. Pudas, B. Aitchison, M. Kauppinen, D. P. Brown, O. G. Okhotnikov, E. I. Kauppinen, ACS Nano, (2011), 5, 3214. 